\title{
Identification of 11 Differentially Expressed Hub Genes and Their Upstream microRNAs in Nasal-type NK/T-cell Lymphoma Based on Clinical Sample Analysis
}

\author{
Daxia Cai ${ }^{1, ~ *, ~ Y i n g ~ Y a n g ~}{ }^{2}$, Zewei Jiang ${ }^{3}$, Dongmei He $\mathrm{H}^{1, \text { * }}$ \\ ${ }^{1}$ Institute of Hematology, Jinan University, Guangzhou, P.R. China \\ ${ }^{2}$ Health Management Center, The First Affiliated Hospital of Jinan University, Guangzhou, China \\ ${ }^{3}$ Department of Respiratory, The First Affiliated Hospital of Jinan University, Guangzhou, China \\ Email address: \\ thedm@jnu.edu.cn (Dongmei He), tfcdx620@stu2018.jnu.edu.cn (Daxia Cai),305923790@qq.com (Ying Yang), \\ jiangzewei163@163.com (Zewei Jiang) \\ ${ }^{*}$ Corresponding author
}

\section{To cite this article:}

Daxia Cai, Ying Yang, Zewei Jiang, Dongmei He. Identification of 11 Differentially Expressed Hub Genes and Their Upstream microRNAs in Nasal-type NK/T-cell Lymphoma Based on Clinical Sample Analysis. Cancer Research Journal. Vol. 8, No. 4, 2020, pp. 68-81. doi: $10.11648 /$ j.crj. 20200804.13

Received: October 27, 2020; Accepted: November 13, 2020; Published: November 23, 2020

\begin{abstract}
Nasal-type NK/T-cell lymphoma (nasal-type NKTL) is one of the most lethal cancers for. Our study aimed to identify hub differentially expressed genes (DE- genes) and their upstream microRNAs between nasal-type NK/T-cell lymphoma (NKTL) tumor samples and normal nasal tissues through integrated bioinformatics. The 503 DE-genes and 106 DE-miRNAs were identified between NKTL and human normal nasal samples. GO and KEGG analysis were significantly enriched in meiotic recombination, regulation of syncytium formation by plasma membrane fusion, deubiquitination, enriched in meiotic recombination, regulation of syncytium formation by plasma membrane fusion, and stem cell division. And 11 differential expression hub genes and their upstream microRNAs were identified between nasal-type NKTL and normal nasal samples. In summary, after a series of analyses, we found that 11 hub DE-genes and their upstream DE-miRNAs (CDC27miR-548c-3p, FREM2- miR-373*, ARHGAP29-miR-548c-3p, QSER1-miR-548c-3p, CD3EAP-miR-149*, SF3A1miR-548c-3p, AQP4-miR-29b, ZFP36L2-miR-142-3p, SRP72-miR-16, TSC22D2-miR-16, TSC22D2-let-7f, DOCK5-miR-16) between nasal-type NKTL and normal nasal samples. They are highly likely to be serve as promising biomarkers in nasal-type NKTL.
\end{abstract}

Keywords: Bioinformatics Analysis, Differentially Expressed Genes (DE-genes),

Differentially Expressed microRNAs (DE-miRNAs), Nasal-type NK/T-cell Lymphoma (nasal-type NKTL), microRNAs

\section{Introduction}

Non-Hodgkin lymphoma (NHL) is a highly heterogeneous proliferative disease that can further develop into B cell lymphoma (accounting for $80 \%-85 \%$ of NHL), and natural killer/T-cell lymphoma (accounting for $15 \%-20 \%$ of NHL). Nasal-type NKTL is a rare subtype of mature T- and NK-cell lymphoma leading to palatal deformity caused by cancer invasion through the hard palate, which accounts for approximately $10 \%$ of all peripheral NK/T-cell lymphomas in America [1]. Although the use of CCRT with platinum or
L-asparaginase-containing chemotherapy and the avoidance of anthracyclines can improve the prognosis of NKTL, patients still have a poor median survival time of less than 2 years according to global NKTL patient statistics [2]. More unfortunately, nearly half of the patients with newly diagnosed NKTL continue to experience disease progression, and the prognosis in patients with relapsed or refractory NKTL remains unsatisfactory [3]. Previous studies have reported that genetic aberrations, the cellular microenvironment, EBV infection, and epigenetic dysregulation are closely related to NKTL occurrence, 
development, and metastasis [4]. A better understanding of the pathogenic mechanisms, especially those of nasal-type NKTL, will provide important insights into the biology of this disease. Therefore, it is urgently necessary to explore the molecular mechanisms underlying nasal-type NKTL occurrence, development, and metastasis to develop targeted therapies and improve prognosis.

MicroRNAs (miRNAs) are a class of noncoding single-stranded RNA molecules with hairpin structures of approximately 22 nucleotides that are involved in regulating the expression of protein-coding genes [5]. At the same time, miRNA regulates gene expression at the posttranscriptional level by pairing with the 3'-untranslated region (3'UTR) of the target gene mRNA. Each miRNA can have multiple target genes and several miRNAs can regulate the same gene. In recent decades, miRNAs have been considered to be involved in the regulation of all aspects of cancers, including proliferation, differentiation and metabolism [6]. However, few studies based on large-scale human tissue studies of differentially expressed miRNAs focused on nasal-type NKTL have been reported.

As gene-chip and RNA sequencing technology are widely used to identify genomic changes during tumour progression, the Gene Expression Omnibus (GEO) has played an important role in bioinformatics analysis. This provides an efficient tool to discover differentially expressed genes (DE-genes) and differentially expressed miRNAs (DE-miRNAs). Based on the GEO database and comprehensive bioinformatics analysis, this study focused on exploring the DE-genes in nasal-type NKTL and their upstream miRNAs, as well as the possible molecular mechanisms in the development of nasal type NKTL compared with normal human nasal tissues. Therefore, we mined and analysed the data from multiple datasets in the GEO database to highlight the characteristics of gene expression and their upstream miRNAs and provide a basis for further research in human nasal-type NKTL.

\section{Materials and Methods}

\subsection{Microarrays and Screening for Differentially Expressed Genes and Differentially Expressed miRNAs}

We logged in to the National Center for Biotechnology Information (NCBI) GEO database (https://www.ncbi.nlm.nih.gov/geo) to search for the microarrays we needed. Then, to ensure the rigor and accuracy of our research, only datasets comparing mRNA/miRNA expression in human nasal-type NKTL samples with that in human normal nasal samples were included. Carefully read the complete information of the searched datasets and then selected the most suitable datasets for further analysis. Then, the GSE80631 [7] and GSE31377 [8] datasets were selected in our study. The dataset GSE80631 is a GPL6883 platform (Illumina HumanRef-8 v3.0 expression beadchip)-based dataset. We selected 13 human normal nasal samples and 19 human nasal-type
NKTL samples from dataset GSE80631. GSE31377 is based on the platform of GPL8227 (Agilent-019118 Human miRNA Microarray 2.0 G4470B (miRNA ID version)). We selected 2 human normal nasal samples and 17 human nasal-type NKTL samples from dataset the GSE31377.

We used the "LIMMA" from the R package of the bioconductor project to normalize the data. To conduct the differential expression analysis of the miRNAs and genes in nasal-type NKTL samples compared to normal nasal tissues, the related codes were entered into R, and the DE-genes and DE-miRNAs were analysed through the "limma" package in the bioconductor package (http://www. bioconductor.org/). $p$-value $<0.05$ and $|\operatorname{logFC}|>1$ were set as the cut-off criteria for identifying DE-genes and DE-miRNAs. At the same time, volcano plots of the DE-genes and DE-miRNAs were generated by $\mathrm{R}$.

\subsection{Prediction of Target Genes for DE-miRNAs}

The potential target genes of the top 10 most upregulated and downregulated DE-miRNAs were obtained from the overlapping part of the predictive results derived from TargetScanHuman [9] Release 7.2 (http://www.targetscan. org), miRTarBase [10] Release 7.0 (http://mirtarbase.mbc. nctu.edu.tw), and miRDB [11] Version 5.0 (http://mirdb.org) through calculating and drawing custom Venn diagrams in the web tool Bioinformatics \& Systems Biology (http://bioinformatics.psb.ugent.be/webtools/Venn/). The TargetScanHuman Release 7.2, which was updated on March 2018 , is a web server that predicts the biological targets of miRNAs by searching for the presence of conserved sites that match human 3'UTRs and the orthologues of each miRNA. The miRTarBase Release 7.0 is an experimentally validated microRNA-target interactions database, which is updated on September 15, 2017. miRDB Version 5.0 was released in August 2014, and is an online database for miRNA target prediction and functional annotations.

\subsection{Enrichment Analysis}

Metascape3.0 (http://metascape.org) [12] was introduced to perform functional and pathway enrichment analysis of the DE-genes and the predicted target genes of the selected top 10 upregulated DE-miRNAs and top 10 downregulated DE-miRNAs, including determination of the Gene Ontology (GO) terms for the biological process (BP), cellular component (CC) and molecular function (MF) categories, Reactome Gene Sets, Canonical Pathways, CORUM, and Kyoto Encyclopedia of Genes and Genomes (KEGG) pathways.

\subsection{Conjoint Analysis of DE-genes and DE-miRNAs}

Due to inhibition by miRNAs of posttranscriptional gene expression via their specific binding to target messenger RNA (mRNA), 10 upregulated DE-genes were crossed with the predicted target genes of the top 10 downregulated DE-miRNAs, and 10 downregulated DE-genes were crossed with the predicted target genes of the top 10 upregulated 
DE-miRNAs by calculating and drawing custom Venn diagrams in the web tool Bioinformatics \& Systems Biology to obtain more convincing DE-miRNAs, DE-genes, and the one-to-one relationships.

\section{Results}

\subsection{Identification of Differentially Expressed Genes and miRNAs}

To identify DE-miRNAs and DE-genes between nasal-type NKTL patient samples and normal nasal tissues, the GSE80631 and GSE31377 data were normalized and differential expression analysis was conducted using the "limma" software package. Then, visualization of normalized data is shown in Figure 1. Based on this differential expression analysis and our screening criteria ( $p$-value $<0.05$, $|\operatorname{logFC}|>1)$, a total of 503 genes were found to be significantly differentially expressed in nasal-type NKTL samples when compared to normal nasal tissues, including 281 upregulated and 222 downregulated genes. At the same time, 106 significantly DE-miRNAs were discovered, of which 57 were upregulated and 49 were downregulated. Furthermore, the top 20 upregulated DE-genes and downregulated DE-genes (Appendix Table 3), downregulated DE-miRNAs and upregulated DE-miRNAs (Appendix Table 4) were ranked by $|\operatorname{logFC}|$ in Appendix Tables 3-4.

Table 1. More convinced upregulated DE-genes and their upstream miRNAs.

\begin{tabular}{lll}
\hline upregulated DE-genes & downregulated DE-miRNAs & $\log \mathbf{C C}$ \\
\hline CDC27 & hsa-miR-548c-3p & 2.41476 \\
FREM2 & hsa-miR-373* & 2.235638 \\
ARHGAP29 & hsa-miR-548c-3p & 2.001139 \\
QSER1 & hsa-miR-548c-3p & 1.802661 \\
CD3EAP & hsa-miR-149* & 1.77193 \\
SF3A1 & hsa-miR-548c-3p & 1.6008 \\
\hline
\end{tabular}

$\mathrm{DE}=$ differentially expressed. *represents immature miRNAs.

Table 2. More convinced downregulated DE- genes and their upstream miRNAs.

\begin{tabular}{lll}
\hline downregulated DE-genes & upregulated DE-miRNAs & $\operatorname{logFC}$ \\
\hline AQP4 & hsa-miR-29b & -2.10407 \\
ZFP36L2 & hsa-miR-142-3p & -1.93154 \\
SRP72 & hsa-miR-16 & -1.54929 \\
TSC22D2 & hsa-let-7f & -1.48424 \\
TSC22D2 & hsa-miR-16 & -1.48424 \\
DOCK5 & hsa-miR-16 & -1.09845 \\
\hline
\end{tabular}

$\mathrm{DE}=$ differentially expressed.

A

B

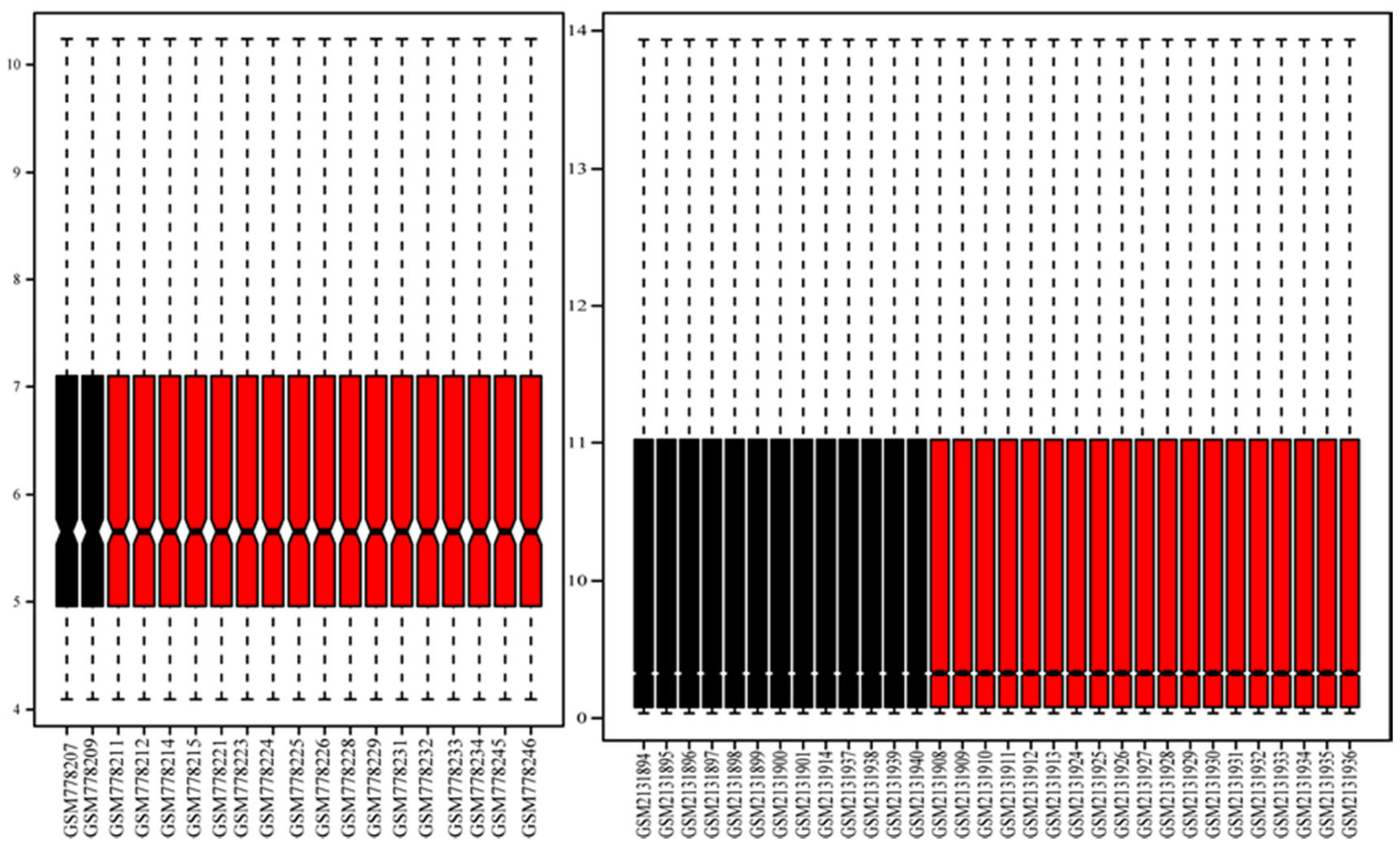

Figure 1. Normalization of datasets GSE31377 (A) and GSE80631 (B).

\subsection{Prediction of Target Genes of DE-miRNAs}

Based on the screening of DE-miRNAs in the previous step, the top 10 DE-miRNAs were selected for target gene prediction. The top 10 upregulated DE-miRNAs were hsa-miR-21, hsa-miR-142-3p, hsa-let-7f, hsa-miR-16, hsa-miR-29a, hsa-let-7g, hsa-miR-142-5p, hsa-miR-20a, hsa-miR-29b, and hsa-miR-451. The top 10 downregulated 
DE-miRNAs were hsa-miR-1225-5p, hsa-miR-373*, hsa-miR-647, hsa-miR-483-3p, hsa-miR-485-3p, hsa-miR-498, hsa-miR-1224-3p, hsa-miR-149*, hsa-miR-548c-3p, and hsa-miR-1224-5p. We searched for the above target genes of miRNAs in the databases miRTarBase, TargetScanHuman and miRDB. The genes predicted by the three databases were identified as target genes for DE-miRNAs. Finally, 1207 miRNA targets were predicted by three databases as the targets of top the 10 upregulated DE-miRNAs (Appendix Table 5). In the same way, 564 miRNA targets were identified as the targets of the top 10 downregulated DE-miRNAs (Appendix Table 6).

\subsection{Pathway and Process Enrichment Analysis}

Through Metascape3.0, enrichment analysis was carried out for DE-genes and the predicted target genes of DE-miRNAs. The networks of enriched terms were visualized using Cytoscape (Figures 2 and 3), where each node represents an enriched term and is coloured first by its cluster ID (Figures 2A1, B1, 3A1 and B1) and then by its $p$-value (Figures 2A2, B2, 3A2 and B2). More details on the pathway and process enrichment analyses are shown in Appendix Tables 7-8. The top 5 results for the pathway and process enrichment analyses for the upregulated DE-genes included meiotic recombination, regulation of syncytium formation by plasma membranes, cytokine-cytokine receptor interaction, and deubiquitination. Only the regulation of syncytium formation by plasma membranes belongs to GO biological processes, while the others belong to the KEGG pathway category. The top 20 clusters of significantly enriched terms for the upregulated DE-genes are shown in Figure 2A1 and A2. The top 5 pathway and process enrichment analyses for predicted target genes of the top 10 downregulated DE-miRNAs included covalent chromatin modification, pathways in cancer, intracellular signalling by second messengers, regulation of cellular amide metabolic process, and protein deacetylation. Except for pathways in cancer (KEGG pathway category) and intracellular signalling by second messengers (Reactome Gene Sets category), all above results belong to the GO biological processes category. The top 20 clusters of significantly enriched terms for predicted target genes of the top 10 downregulated DE-miRNAs are shown in Figure $2 \mathrm{~B} 1$ and B2. The top 5 pathway and process enrichment analyses for the downregulated DE-genes included divalent metal ion transport, intracellular mRNA localization, hair follicle maturation, stem cell division, and the ERK1 and ERK1 cascades. They all belong to the GO biological processes category. There were only 17 clusters of significantly enriched terms for downregulated DE-genes; therefore, only 17 clusters are shown in Figure $3 \mathrm{~A} 1$ and A2. The top 5 pathway and process enrichment analyses for 10 predicted target genes of upregulated DE-miRNAs included heart development, blood vessel development, muscle structure development, pathways in cancer, and response to growth factor. Only pathways in cancer belong to the KEGG pathway category, while others belong to GO biological processes. The top 20 clusters of significantly enriched terms for predicted target genes of the 10 upregulated DE-miRNAs are shown in Figure 3B1 and B2.

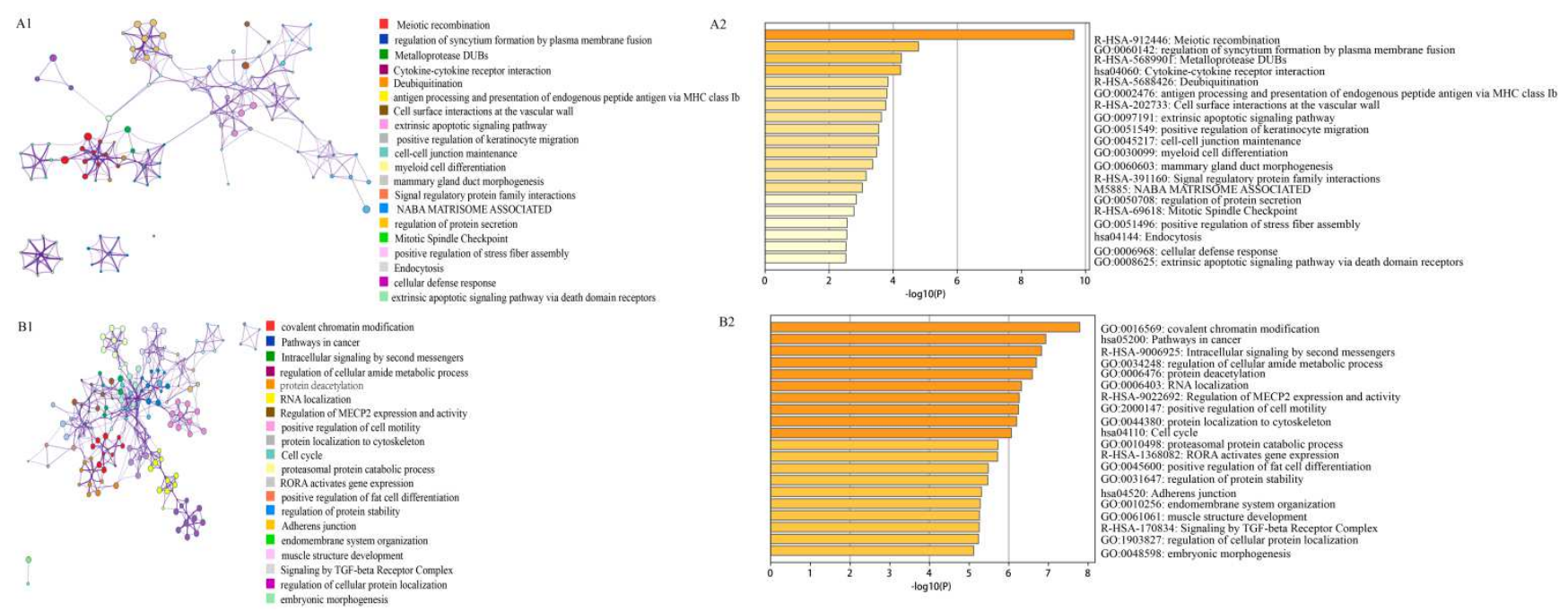

Figure 2. Functional enrichment of upregulated DE-genes and predicted target genes of the top 10 downregulated DE-miRNAs:(A) The visualized networks of enriched upregulated DE-genes, where each node represents an enriched term and is colored first by its cluster ID (A1) and then by its $P$-value (A2). (B) The visualized networks of enriched predicted target genes of top 10 downregulated DE-miRNAs, where each node represents an enriched term and is colored first by its cluster ID (B1) and then by its $P$-value (B2). DE=differentially expressed. 

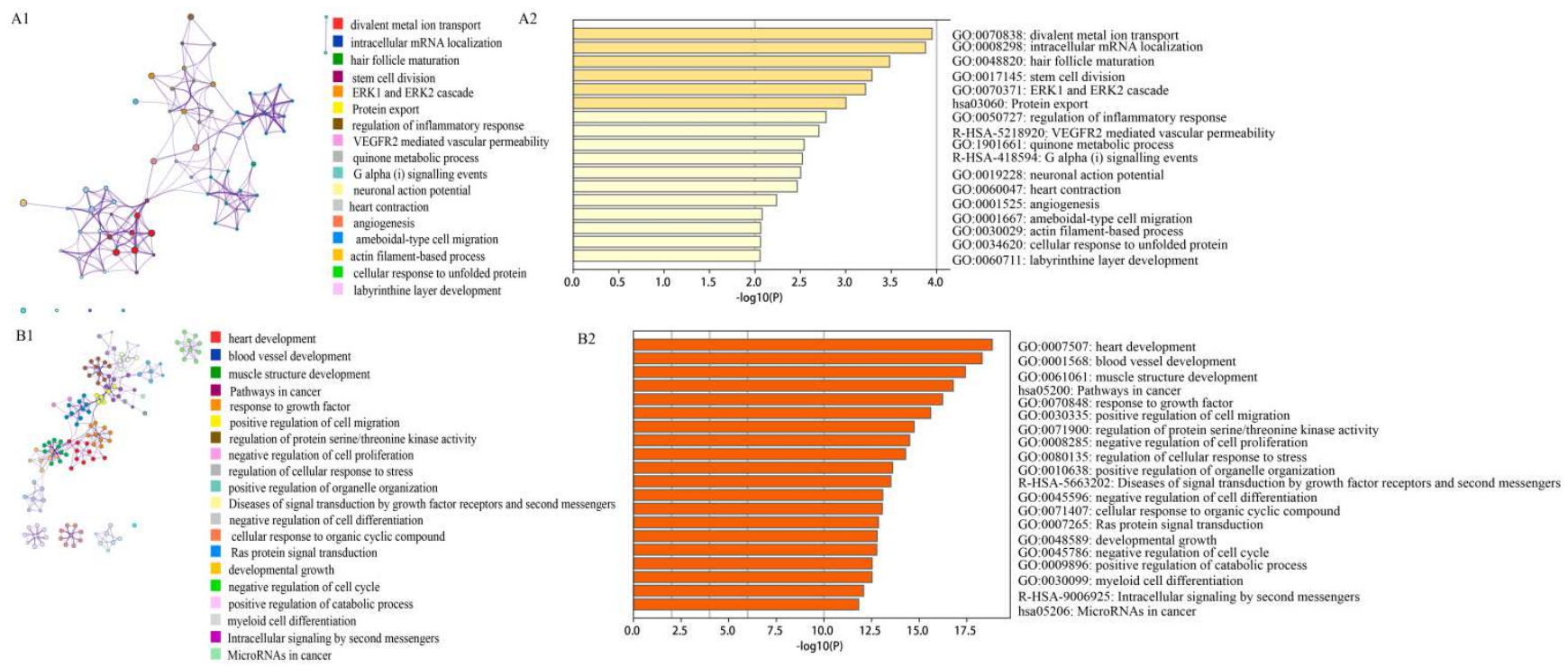

Figure 3. Functional enrichment of downregulated DE-genes and predicted target genes of upregulated DE-miRNAs: (A) The visualized networks of enriched downregulated DE-genes, where each node represents an enriched term and is colored first by its cluster ID (A1) and then by its $P$-value (A2). (B) The visualized networks of enriched target genes of the 10 predicted target genes of upregulated DE-miRNAs, where each node represents an enriched term and is colored first by its cluster ID (B1) and then by its $P$-value (B2). DE=differentially expressed.
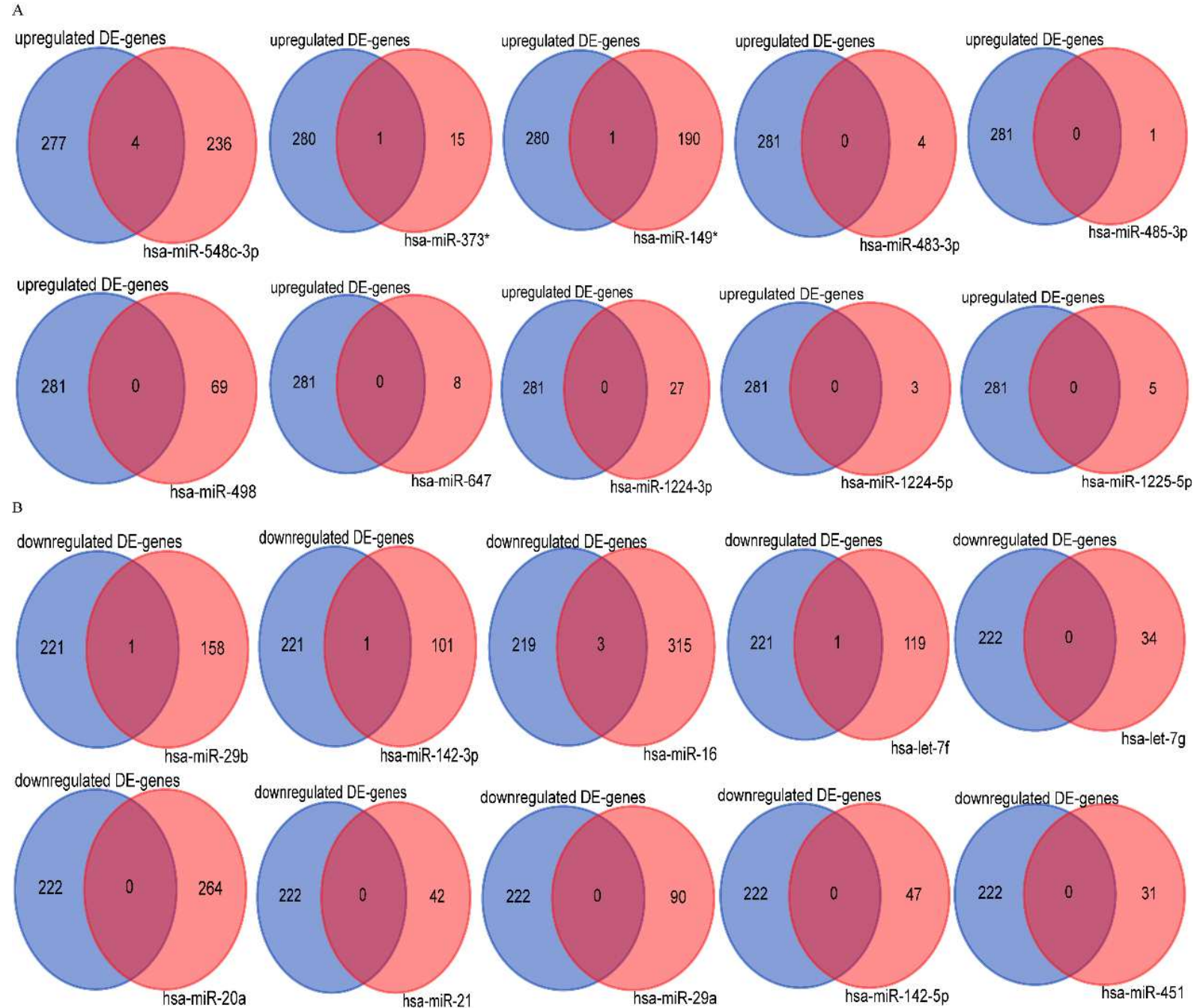

Figure 4. The intersection of upregulated DE-genes and the target genes of downregulated DE-miRNAs (A) and upregulated DE-miRNAs (B). $D E=$ differentially expressed. 


\subsection{Conjoint Analysis of DE-miRNAs and DE-genes}

To connect DE-miRNAs with DE-genes, the predicted target genes of DE-miRNAs were intersected with the DE-genes (Figure 4). In the end, we obtained six upregulated and five downregulated DE-genes and their upstream miRNAs. The upregulated DE-genes and their upstream miRNAs are shown below (Table 1). They were CDC27 (Cell Division Cycle 27)-miR-548c-3p, FREM2 (FRAS1 Related Extracellular Matrix 2)-miR-373*, ARHGAP29 (Rho GTPase Activating Protein 29)-miR-548c-3p, QSER1 (Glutamine and Serine Rich 1)-miR-548c-3p, CD3EAP (CD3e Molecule Associated Protein)-miR-149*, and SF3A1 (Splicing Factor 3a Subunit 1)-miR-548c-3p. At the same time, we found downregulated DE-genes and their upstream miRNAs (Table 2). They were AQP4 (Aquaporin 4)-miR-29b, ZFP36L2 (ZFP36 Ring Finger Protein Like 2)-miR-142-3p, SRP72 (Signal Recognition Particle 72)-miR-16, TSC22D2 (TSC22 Domain Family Member 2)-miR-16, TSC22D2-let-7f, and DOCK5 (Dedicator of Cytokinesis 5)-miR-16.

\section{Discussion}

The treatment of patients with NKTL has improved over the past decades. Gene therapy is increasingly becoming an effective way to treat cancer based on the identification of DE-genes in human disease. Abnormal expression of miRNAs is involved in regulating all aspects of the occurrence, invasion and metastasis of cancers, which play an important role in cancer diagnosis and treatment [6]. However, until now, DE-genes and DE-miRNAs focused on nasal-type NKTL compared with normal nasal NKTL have rarely been reported. Fortunately, the integration of bioinformatics and microarray technology has enabled more effective ways to identify DE-genes and DE-miRNAs, which can help to discover potential effective targets for the diagnosis and treatment of nasal-type NKTL patients. It is necessary to further study the functions of miRNAs, predict their target genes and understand the interaction between miRNAs and target genes in nasal-type NKTL at present.

In the present study, we screened an eligible gene dataset and miRNA dataset in the GEO database and obtained DE-genes and DE-miRNAs after standardized processing. A total of 503 significantly DE-genes were discovered, of which 281 were upregulated and 222 were downregulated. Furthermore, 106 miRNAs were screened out to be significantly differentially expressed in human nasal-type NKTL samples when compared to human normal nasal samples, including 57 upregulated and 49 downregulated miRNAs. A total of 1207 miRNA targets were predicted for the top 10 upregulated DE-miRNAs, and 564 miRNA targets were identified for the top 10 downregulated DE-miRNAs. At last, the one-to-one relationship between DE-genes and DE-miRNAs were obtained. Through enrichment analysis, we found that meiotic recombination, regulation of syncytium formation by plasma membrane fusion, deubiquitination, cytokine-cytokine receptor interaction, divalent metal ion transport, intracellular mRNA localization, hair follicle maturation, ERK1 and ERK2 cascade, and stem cell division were more likely to be involved in the development of nasal-type NKTL. Meiotic recombination events have been implicated in cancer development, including ovarian carcinomas, lung adenocarcinoma, head and neck squamous cell carcinoma, liver cancer, bladder urothelial carcinoma, and breast cancer, but meiotic recombination events in nasal-type NKTL have not yet been reported [13]. For nasal-type NKTL, regulation of syncytium formation by plasma membrane fusion is thought to be related to the Epstein-Barr virus (EBV) entry mechanism, which is useful for the development of infection inhibitors and developing EBV vaccine approaches [14]. Metalloprotease deubiquitinases can regulate the ubiquitin signalling cascade, which are thought to be new regulators in cancer progression, such as non-small cell lung cancer [15], but their involvement in nasal-type NKTL has not been previously reported.

In our study, six upregulated and five downregulated DE-genes and their upstream miRNAs were identified by analysing the one-to-one relationship between DE-genes and DE-miRNAs. CDC27-miR-548c-3p, FREM2-miR-373 ${ }^{*}$ and ARHGAP29-miR-548c-3p were the top 3 upregulated genes and their upstream miRNAs in the nasal-type NKTL samples compared with normal nasal tissues. CDC27 (also known as ANAPC3) was recently reported to be related to tumour progression and poor survival in T-cell lymphoblastic lymphoma [16]. Additionally, FREM2 has been found to play an important role in the malignant progression of prostate adenocarcinoma [17], and it is also thought to be a biomarker of glioblastoma stem cells [18]. It has been demonstrated that miR- $373^{*}$ can inhibit cell proliferation in colon cancer [19], and downregulation of miR-373* was confirmed in childhood B-cell precursor acute lymphoblastic leukaemia [20]. ARHGAP29 is involved in the development and migration of tumors. Moreover, Margit Schraders et al. demonstrated that ARHGAP29 may be involved in mantle cell lymphoma development and maintenance through signal transduction [21]. There are few reports focused on CDC27, FREM2, ARHGAP29, QSER1, CD3EAP, SF3A1, miR-548c-3p, miR-373* ${ }^{*}$ miR-548c-3p, miR-548c-3p, miR-149* and miR-548c-3p in nasal-type NKTL, and we believe these are worthy of further research.

In the present study, five downregulated DE-genes and their upstream miRNAs were identified (Table 2). AQP4 (miR-29b), ZFP36L2 (miR-142-3p) and SRP72 (miR-16) were the top 3 downregulated genes (upstream miRNAs) in nasal-type NKTL samples compared with normal nasal tissues. MicroRNA-29b is downregulated in cerebral ischaemia and participates in the occurrence and development of cerebral ischaemia by targeting AQP4 regulation [22]. Furthermore, AQP4 induces paraneoplastic syndrome by antibody production, which may be the mechanism of acute myelogenous leukaemia coexisting with 
optic neuritis after transplantation [23]. At the same time, AQP4 has been demonstrated to be involved in cell proliferation, invasiveness, migration, and apoptotic potential in glioma cells [24]. miR-29b has been reported to promote the occurrence and development of acute myeloid leukaemia [25] by participating in the regulation of tumour cell proliferation and apoptosis. Repression of miR-29b participates in regulation in cutaneous T-cell lymphoma through the miR-29b-BRD4 axis [26]. Moreover, it was demonstrated that miR-29s could alter the expression of targets involved in critical cancer pathways in Burkitt's lymphoma, such as cell cycle control, apoptosis inhibition and DNA methylation [27]. Moreover, T lymphoma invasion and metastasis 1 (Tiam1) was identified as a direct target of miR-29b [28]. However, so far, we have not found reports on miR-29b focused on nasal-type NKTL, which may become the innovation point and hot spot of future research. ZFP36L2 (also known as TIS11D, one of the NOTCH RNA repressors), dysfunction is associated with the pathogenesis of certain types of leukaemia, such as acute myeloid leukaemia and Burkitt's lymphoma [29]. In addition, ZFP36L2 plays a role in the development of acute lymphoblastic leukaemia [30]. However, there is little literature focused on the relationship between these genes and microRNAs (AQP4, ZFP36L2, SRP72, TSC22D2, DOCK5, miR-29b, miR-142-3p, miR-16, and let-7f) in nasal-type NKTL patients, which may deserve more in-depth research.

In our study, CDC27, ARHGAP29, QSER1, SF3A1 (miR-548c-3p), FREM2- miR-373* , CD3EAP-miR-149*, AQP4-miR-29b, ZFP36L2-miR-142-3p, SRP72, TSC22D2, DOCK5 (miR-16), and TSC22D2-let-7f were identified between nasal-type NKTL and normal nasal samples. Among them, eleven differentially expressed genes (CDC27, FREM2, ARHGAP29, QSER1, CD3EAP, SF3A1, AQP4, ZFP36L2, SRP72, TSC22D2, DOCK5) and six differentially expressed miRNAs (miR-548c-3p, miR-373* , miR-149*, miR-29b, miR-142-3p, let-7f) have been confirmed to be associated with other types of lymphoma, other cancers, or other non-neoplastic diseases. However, few DE-genes and DE-miRNAs focused on nasal-type NKTL have been explored, which may be an innovation of future research. The DE-genes and DE-miRNAs can serve as biomarkers in nasal-type NKTL, which is helpful to understand the pathogenesis of nasal-type NKTL patients. In addition, it may provide strong theoretical support for the early diagnosis of nasal-type NKTL patients and the development of novel therapeutic targets. However, it still has limitations:

1). Target gene prediction was only carried out for the top 10 DE-miRNAs;

2). Only the target genes of the top 10 DE-miRNAs were selected for further enrichment analysis;
3). Since there have been few studies focused on nasal-type NKTL, no dataset with a larger sample size can be found, so, 2 human normal nasal samples and 17 human nasal-type NKTL samples from the GSE313772 dataset were chosen in our study.

4). Due to a lack of experimental verification, more research should be carried out.

\section{Conclusions}

In conclusion, we successfully identified DE-genes and their upstream DE-miRNAs (CDC27- miR-548c-3p, FREM2- $\quad$ miR-373* $\quad$ ARHGAP29-miR-548c-3p, QSER1-miR-548c-3p, CD3EAP-miR-149*, SF3A1miR-548c-3p, AQP4-miR-29b, ZFP36L2-miR-142-3p, SRP72-miR-16, TSC22D2-miR-16, TSC22D2-let-7f, and DOCK5-miR-16) in nasal-type NKTL based on clinical sample tissues through bioinformatic analysis, and these molecules are highly likely to be related to nasal-type NKTL and which may serve as biomarkers in nasal-type NKTL. Undoubtedly, continued efforts to delineate the mechanism of differential genes and miRNAs will uncover novel insights into nasal-type NKTL.

\section{Abbreviations}

differentially expressed genes (DE-genes), differentially expressed microRNAs (DE-miRNAs), nasal-type NK/T-cell lymphoma (nasal-type NKTL), Cell Division Cycle 27 (CDC27), FRAS1 Related Extracellular Matrix 2 (FREM2), Rho GTPase Activating Protein 29 (ARHGAP29), Glutamine And Serine Rich 1 (QSER1), CD3e Molecule Associated Protein (CD3EAP), Splicing Factor 3a Subunit 1 (SF3A1), Aquaporin 4 (AQP4), ZFP36 Ring Finger Protein Like 2 (ZFP36L2), Signal Recognition Particle 72 (SRP72), TSC22 Domain Family Member 2 (TSC22D2), Dedicator Of Cytokinesis 5 (DOCK5)

\section{Formatting of Funding Sources}

Funding: This research did not receive any specific grant from funding agencies in the public, commercial, or not-for-profit sectors.

\section{Acknowledgements}

The project was supported by Overseas Chinese Affairs Office of the State Council Key Discipline Construction Fund (51205002).

\section{Appendix}

Table 3. The top 20 upregulated and downregulated DE-genes (ranked by $|\log F C|$ ).

\begin{tabular}{lllll}
\hline Genes & logFC & "t" & "P.Value" & "adj.P.Val" \\
\hline UBAP2L & 3.879406091 & 7.711088382 & $8.31 \mathrm{E}-09$ & expression \\
TNFRSF12A & 3.65190092 & 5.122697428 & $1.37 \mathrm{E}-05$ & upregulation \\
\hline
\end{tabular}




\begin{tabular}{|c|c|c|c|c|c|}
\hline Genes & $\log \mathrm{FC}$ & "t" & "P.Value" & "adj.P.Val" & expression \\
\hline HIST1H4J & 3.452632998 & 4.705086766 & $4.62 \mathrm{E}-05$ & 0.004879843 & upregulation \\
\hline SCTR & 3.320035717 & 3.577358316 & 0.00112315 & 0.026257114 & upregulation \\
\hline CETN2 & 3.016183112 & 4.667267489 & $5.16 \mathrm{E}-05$ & 0.005020758 & upregulation \\
\hline ADAMTS20 & 2.944247403 & 3.369083932 & 0.001971425 & 0.035561324 & upregulation \\
\hline LOC401565 & 2.931236947 & 4.673618746 & $5.06 \mathrm{E}-05$ & 0.005020758 & upregulation \\
\hline TGFBR1 & 2.905484306 & 6.19925479 & $5.97 \mathrm{E}-07$ & 0.000400622 & upregulation \\
\hline FAM3D & 2.903349382 & 5.320153275 & 7.71E-06 & 0.001766605 & upregulation \\
\hline CD177 & 2.85437046 & 3.256806836 & 0.002656712 & 0.041405943 & upregulation \\
\hline HIST2H2AC & 2.847568264 & 4.558287141 & 7.07E-05 & 0.006279004 & upregulation \\
\hline DOK5 & 2.818048072 & 3.382679659 & 0.001901008 & 0.035099561 & upregulation \\
\hline MGC13017 & 2.815718644 & 5.604320752 & 3.36E-06 & 0.001022668 & upregulation \\
\hline IL26 & 2.763884207 & 4.661045869 & $5.25 \mathrm{E}-05$ & 0.005020758 & upregulation \\
\hline IL25 & 2.750375101 & 4.404942125 & 0.000109875 & 0.007882469 & upregulation \\
\hline HIST1H2BF & 2.747516007 & 3.910023591 & 0.000447662 & 0.015875964 & upregulation \\
\hline SIRPG & 2.736534779 & 5.276810906 & $8.75 \mathrm{E}-06$ & 0.001868193 & upregulation \\
\hline SLC9A3R2 & 2.724778021 & 3.518571975 & 0.001317952 & 0.028738072 & upregulation \\
\hline CSF2RB & 2.681546694 & 3.719856346 & 0.000759591 & 0.020481387 & upregulation \\
\hline HIST1H2BB & 2.632406983 & 4.114378133 & 0.000251746 & 0.011829561 & upregulation \\
\hline KLF4 & -3.29919148 & -4.15919297 & 0.000221693 & 0.010960098 & downregulation \\
\hline SYN3 & -3.11937921 & -4.10098413 & 0.00026148 & 0.011929085 & downregulation \\
\hline ZNF207 & -3.03922233 & -5.26117862 & $9.15 \mathrm{E}-06$ & 0.001911965 & downregulation \\
\hline KRTHB4 & -2.99265976 & -4.36352981 & 0.000123736 & 0.008075495 & downregulation \\
\hline ZFP36L1 & -2.95271722 & -4.39633941 & 0.000112622 & 0.007920838 & downregulation \\
\hline SLN & -2.94525138 & -3.53752539 & 0.001251829 & 0.027826839 & downregulation \\
\hline ZBTB16 & -2.89774713 & -3.91918127 & 0.00043633 & 0.015750361 & downregulation \\
\hline FGF18 & -2.86338653 & -4.5475020 & 7.29E-05 & 0.006279004 & downregulation \\
\hline AMOTL2 & -2.85713112 & -6.34292943 & $3.94 \mathrm{E}-07$ & 0.000320764 & downregulation \\
\hline NPBWR1 & -2.80828507 & -6.69978677 & $1.42 \mathrm{E}-07$ & $1.90 \mathrm{E}-04$ & downregulation \\
\hline EFHA2 & -2.71571717 & -7.24136302 & $3.06 \mathrm{E}-08$ & $9.59 \mathrm{E}-05$ & downregulation \\
\hline ZNF599 & -2.67703321 & -4.66520744 & 5.19E-05 & 0.005020758 & downregulation \\
\hline ZNF471 & -2.66268816 & -4.09394917 & 0.00026674 & 0.011994354 & downregulation \\
\hline TCAP & -2.63710513 & -4.04257123 & 0.000308425 & 0.013175341 & downregulation \\
\hline ZFP1 & -2.59412544 & -5.42330123 & 5.70E-06 & 0.001531354 & downregulation \\
\hline ZNF503 & -2.59296641 & -4.74399366 & 4.13E-05 & 0.004731436 & downregulation \\
\hline RRBP1 & 0.621289108 & 3.768999144 & 0.000663049 & 0.019889056 & downregulation \\
\hline S100PBP & -2.49782697 & -4.26233293 & 0.000165278 & 0.009319239 & downregulation \\
\hline SUSD4 & -2.49727748 & -4.21461472 & 0.000189365 & 0.010054514 & downregulation \\
\hline FAM100B & -2.48087708 & -4.29613737 & 0.000150065 & 0.009099835 & downregulation \\
\hline
\end{tabular}

$\mathrm{DE}=$ differentially expressed.

Table 4. The top 20 downregulated and upregulated DE-miRNAs (ranked by $|\log F C|$ ).

\begin{tabular}{|c|c|c|c|c|}
\hline miRNA_ID & adj.P.Val & P.Value & $\log \mathrm{FC}$ & expression \\
\hline hsa-miR-1225-5p & 0.04203 & 0.0010752 & -3.86404 & downregulation \\
\hline hsa-miR-373* & 0.04109 & 0.0006506 & -3.74013 & downregulation \\
\hline hsa-miR-647 & 0.03523 & 0.0003004 & -3.48445 & downregulation \\
\hline hsa-miR-483-3p & 0.04203 & 0.0009398 & -3.36585 & downregulation \\
\hline hsa-miR-485-3p & 0.04109 & 0.0006485 & -3.35861 & downregulation \\
\hline hsa-miR-498 & 0.04203 & 0.0009074 & -3.35428 & downregulation \\
\hline hsa-miR-1224-3p & 0.04203 & 0.0007327 & -3.1557 & downregulation \\
\hline hsa-miR-149* & 0.12347 & 0.0091741 & -3.05849 & downregulation \\
\hline hsa-miR-548c-3p & 0.04203 & 0.0008541 & -3.05768 & downregulation \\
\hline hsa-miR-1224-5p & 0.22838 & 0.0317172 & -2.98012 & downregulation \\
\hline hsa-miR-300 & 0.04109 & 0.0005342 & -2.76202 & downregulation \\
\hline hsa-miR-617 & 0.10631 & 0.0071221 & -2.68626 & downregulation \\
\hline hsa-miR-520b & 0.0708 & 0.0030183 & -2.65173 & downregulation \\
\hline hsa-miR-188-5p & 0.18665 & 0.020006 & -2.6094 & downregulation \\
\hline hsa-miR-371-5p & 0.26757 & 0.0456272 & -2.48873 & downregulation \\
\hline hsa-miR-557 & 0.22838 & 0.0319724 & -2.3795 & downregulation \\
\hline hsa-miR-609 & 0.06622 & 0.0027426 & -2.32371 & downregulation \\
\hline hsa-miR-423-5p & 0.03811 & 0.0003713 & -2.26901 & downregulation \\
\hline hsa-miR-939 & 0.19316 & 0.0216456 & -2.15537 & downregulation \\
\hline hsa-miR-574-5p & 0.26757 & 0.0453523 & -2.14901 & downregulation \\
\hline hsa-miR-21 & 0.00861 & $2.097 \mathrm{E}-05$ & 6.24605 & upregulation \\
\hline hsa-miR-142-3p & 0.05704 & 0.0018809 & 4.739127 & upregulation \\
\hline hsa-let-7f & 0.10047 & 0.0064858 & 3.887418 & upregulation \\
\hline hsa-miR-16 & 0.06261 & 0.0025167 & 3.842062 & upregulation \\
\hline
\end{tabular}




\begin{tabular}{lllll}
\hline miRNA_ID & adj.P.Val & P.Value & logFC & expression \\
\hline hsa-miR-29a & 0.09996 & 0.0062097 & 3.812272 & upregulation \\
hsa-let-7g & 0.14563 & 0.0120444 & 3.667266 & upregulation \\
hsa-miR-142-5p & 0.05704 & 0.0020843 & 3.48577 & upregulation \\
hsa-miR-20a & 0.08195 & 0.0047914 & 3.479459 & upregulation \\
hsa-miR-29b & 0.07965 & 0.0045596 & 3.479448 & upregulation \\
hsa-miR-451 & 0.14935 & 0.0125544 & 3.47876 & upregulation \\
hsa-miR-342-3p & 0.07219 & 0.0031656 & 3.423527 & upregulation \\
ebv-miR-BART14 & 0.14563 & 0.0120354 & 3.351517 & upregulation \\
ebv-miR-BART9 & 0.17942 & 0.0187394 & 3.257656 & upregulation \\
hsa-miR-801 & 0.06261 & 0.0024403 & 3.151849 & upregulation \\
hsa-miR-106b & 0.07854 & 0.0042094 & 3.120468 & upregulation \\
hsa-let-7a & 0.17747 & 0.01759 & 3.114659 & upregulation \\
hsa-miR-15b & 0.17747 & 0.0180421 & 3.104831 & upregulation \\
hsa-miR-26a & 0.24196 & 0.0362505 & 3.081633 & upregulation \\
ebv-miR-BART3 & 0.21875 & 0.0282662 & 3.048915 & upregulation \\
hsa-miR-103 & 0.10743 & 0.0073278 & & 3.018221 \\
\hline
\end{tabular}

$\mathrm{DE}=$ differentially expressed.

Table 5. Target genes of top 10 upregulated differentially expressed miRNAs

\begin{tabular}{|c|c|c|}
\hline & $\mathbf{r}$ & \\
\hline $\begin{array}{l}\text { hsa-miR- } \\
21\end{array}$ & 42 & $\begin{array}{l}\text { TG2, NFIA, PDCD4, FBXO11, SPRY2, TIAM1, PCBP1, HNRNPK, WWP1, CBX4, DAXX, NTF3, COL4A1, RASA1, PIK3R1, } \\
\text { MAP2K3, SATB1, MEF2C, CDC25A, TGFB2, TIMP3, YOD1, SOCS6, DDAH1, TAP1, JAG1, ANKRD46, SMAD7, STAT3, } \\
\text { CCL20, IL12A, TOPORS, FASLG, RTN4, PPARA, BMPR2, SOX5, RASGRP1, NFIB, RECK, MSH2, TGFBI }\end{array}$ \\
\hline hsa-let-7f & 120 & $\begin{array}{l}\text { ZNF799, SOD2, DIP2A, ITGA3, BAZ1B, PPP1R15B, MFSD8, LCOR, IRS1, EPHA4, AHR, MAP2K4, GSK3B, PANK3, ATL1, } \\
\text { KMT2D, TXLNG, C5orf51, KLHL15, PAPOLG, CREB1, PPP2R2A, ZNF585A, ATXN1L, SMARCAD1, ATG12, NRBF2, } \\
\text { HNRNPU, RPS16, IRF2BP2, ZNF609, ARMC8, SMC1A, CCL7, GABRG1, DYRK2, NAA20, STX7, TGFBR1, C1 orf52, } \\
\text { COL4A3BP, CREBZF, TRAPPC10, HMGB2, HSPE1-MOB4, CD59, DUSP6, SP1, MBNL1, TP53INP1, PLAGL2, MSI2, } \\
\text { TRIM71, FAM43A, MLLT10, ZNF800, TOR1AIP2, EIF4A2, GPRIN3, CBX5, PEG10, USP1, ZNF200, PDE12, IGF2BP3, } \\
\text { ZNF181, DLX2, ARIH1, MOB4, BEND4, NR2F2, PRDM1, ZNF608, ZFX, ASB11, SMAD2, HNRNPA2B1, NHLRC2, } \\
\text { TSC22D2, PMAIP1, SHOC2, HAT1, TGFBR3, MXD1, NUDT4, RNF11, SEC24A, EDEM3, PDLIM5, MYADM, ZNF652, } \\
\text { MTFR1, E2F8, MEF2D, WASL, BTG1, CDKN1B, DSG2, CASD1, AASDHPPT, LRIG3, ZNF644, GOLGA7, STX3, PHACTR4, } \\
\text { VEZF1, SYT1, SESN3, SOCS7, RORA, ANAPC16, HDAC2, ACER2, VCL, BACH1, KLF10, SALL3, ONECUT2, STRN, } \\
\text { SNX17 }\end{array}$ \\
\hline $\begin{array}{l}\text { hsa-miR- } \\
16\end{array}$ & 318 & 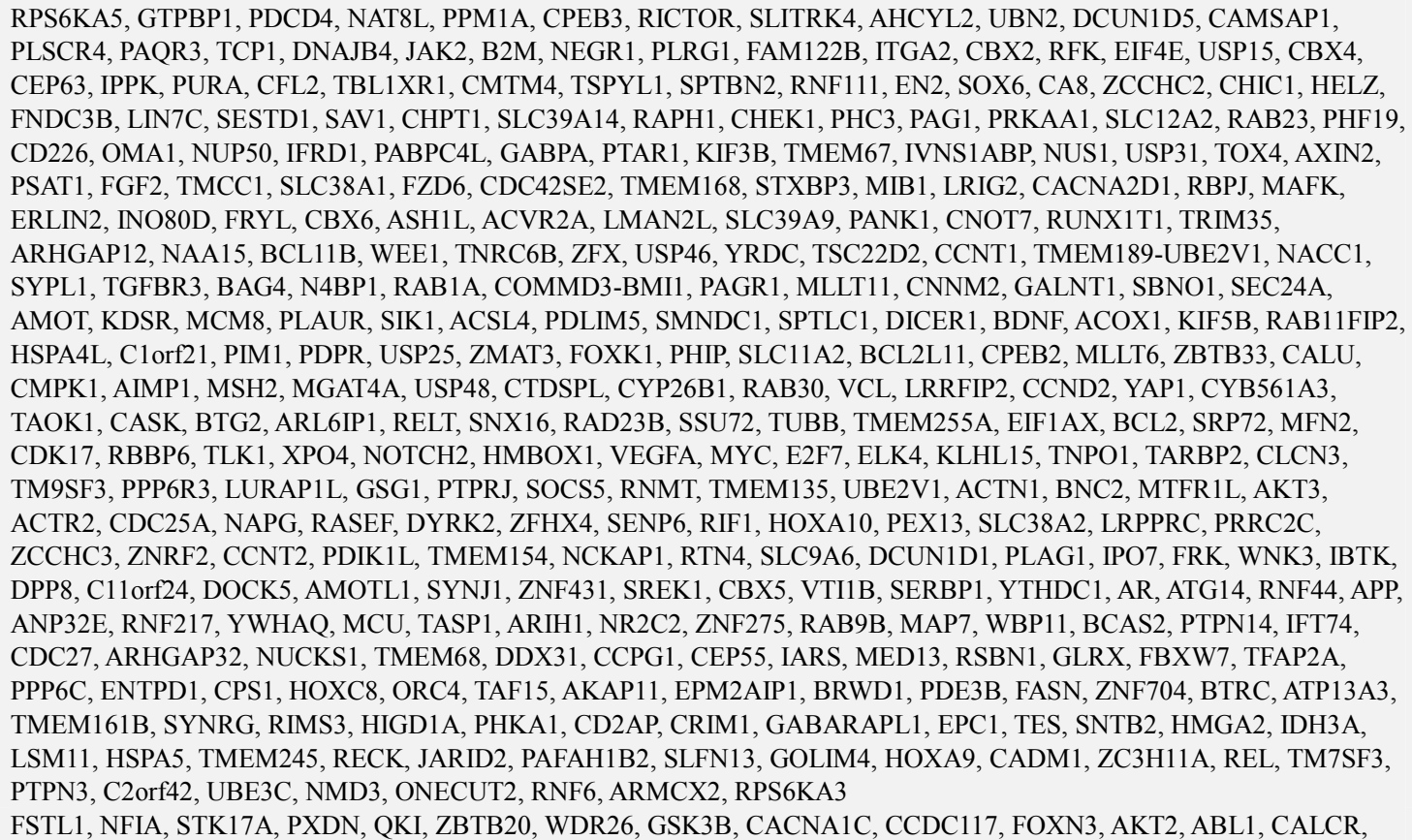 \\
\hline
\end{tabular}




\begin{tabular}{|c|c|c|}
\hline miRNAs & Imber & nes \\
\hline $29 a$ & & $\begin{array}{l}\text { LPL, AAK1, TMOD3, AKT3, HECW1, COMMD2, SH3GLB1, RCC2, HOXA10, ABCE1, ROBO1, CCNT2, IFRD1, CDC7, } \\
\text { SEC31A, AGO1, MAPK6, IYD, ITGA11, WWTR1, FOXO3, ZBTB5, CRKL, ADAM12, IGF1, LILRB2, TET3, BBC3, CAND1, } \\
\text { SERPINB9, DNMT3B, HLF, SOWAHB, COL5A2, DNMT3A, ITGA6, KCNN3, LIMS1, NAA40, HMGCR, KDM5B, PPP3CB, } \\
\text { FSCN1, AMMECR1L, PHACTR2, SYNCRIP, LOX, MXD1, CLDN1, PPM1D, AMER1, PTEN, GPR180, DICER1, REST, } \\
\text { FEM1B, NRIP1, ZCCHC24, EMP1, TMTC3, TECPR2, SLC24A2, FBN1, EPHX2, GOLGA7, BACE1, ZFP91, TET2, GLUL, } \\
\text { RAB30, REL, NEDD9, CCND2, AGAP1, GEM, VHL }\end{array}$ \\
\hline $\begin{array}{l}\text { hsa-let-7 } \\
\text { g }\end{array}$ & 34 & $\begin{array}{l}\text { CCNB1, RAB3IP, KPNA2, ABI2, RDX, KIAA0930, OLR1, ABT1, MBD2, USP38, KMT2D, HAT1, POTEM, AGPAT5, } \\
\text { ZNF704, YOD1, ARID3A, MTUS1, MAP3K1, ABHD17C, HMGB2, HMGA2, CASP3, CLDN12, SYT1, SOCS7, MAPK6, } \\
\text { TNFRSF10B, RAB5C, NAA30, ACER2, CBX5, WAC, APP }\end{array}$ \\
\hline $\begin{array}{l}\text { hsa-miR- } \\
142-5 p\end{array}$ & 47 & $\begin{array}{l}\text { ACTN4, U2SURP, RGMB, PTEN, IGF2BP3, DDX5, RPS6KA5, SAMD12, RHOC, REST, ULK1, ATP13A3, DUSP2, ACTC1, } \\
\text { SLITRK4, HSPA8, ZBTB20, UHMK1, ZBTB43, NFE2L2, YY1, LEPROT, EFCAB14, ROBO1, SLC30A7, FAM107B, LUZP2, } \\
\text { TNS1, PTPN4, SLC24A2, BCOR, FIGN, TRIP10, RGPD4, FEM1C, MED17, CEP97, SOCS1, ZFYVE26, CREBRF, TGFBR2, } \\
\text { ATXN1, LZIC, EGLN3, PTP4A1, AKAP11, MFSD9 }\end{array}$ \\
\hline $\begin{array}{l}\text { hsa-miR- } \\
20 \mathrm{a}\end{array}$ & 264 & 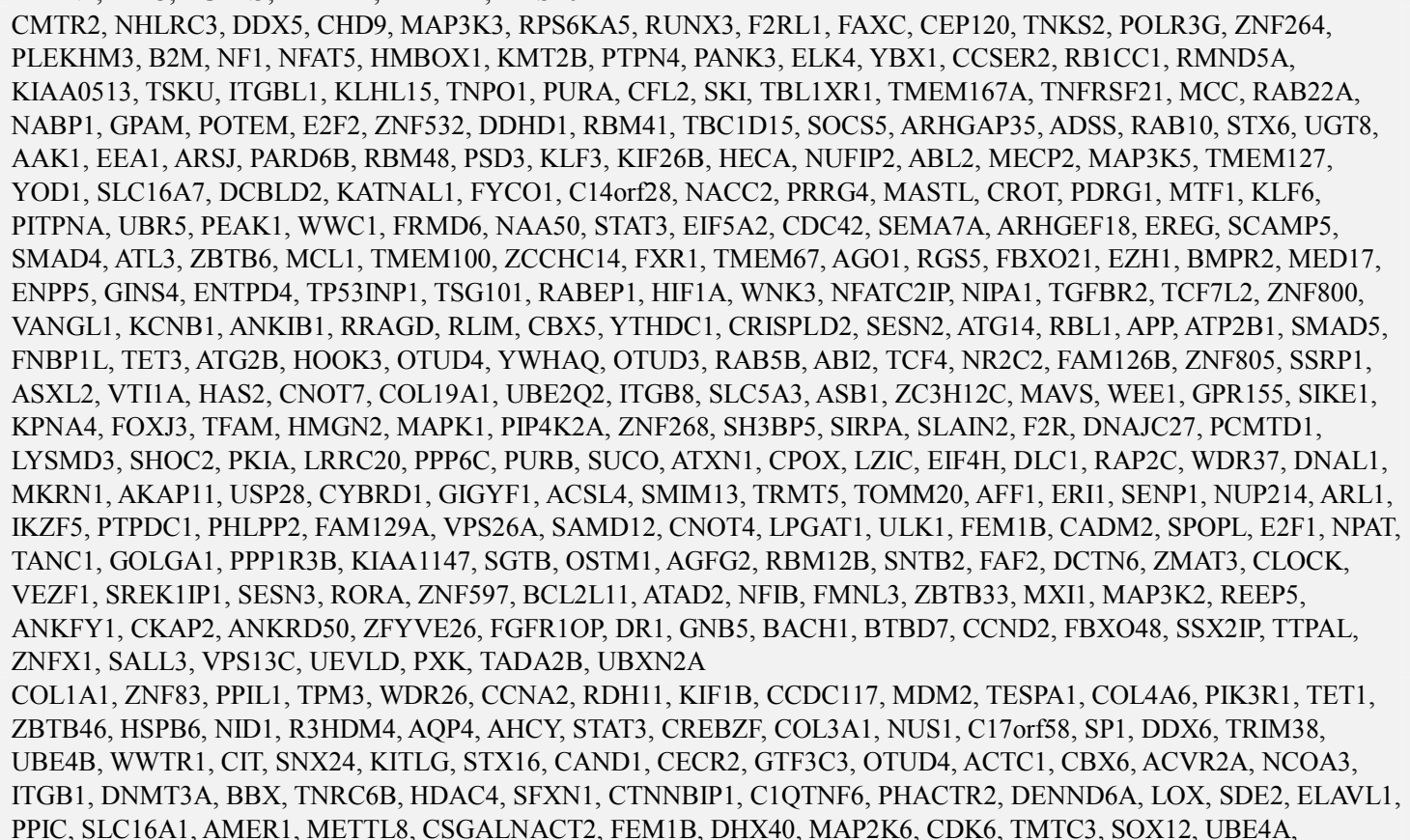 \\
\hline $\begin{array}{l}\text { hsa-miR- } \\
29 \mathrm{~b}\end{array}$ & 159 & $\begin{array}{l}\text { GOLGA7, LOXL2, CALU, TET2, THBS2, SRSF2, CRTC3, NEDD9, CCND2, AMD1, VHL, BTG2, EPB41L4B, PTCD3, } \\
\text { LMNB2, PPP1R15B, KDM2A, HP1BP3, TNRC18, TRAM2, RAB15, NOTCH2, VEGFA, GSK3B, TNFAIP3, PTBP3, TCERG1, } \\
\text { NREP, PDGFRA, AKT3, HECW1, TGFB2, COMMD2, SH3GLB1, FGB, FOLR1, PDGFB, ABCE1, CDC42, NEUROD1, } \\
\text { CCNT2, RNF138, MCL1, PIK3CG, TDG, PLAG1, ZDHHC5, C21orf91, ACVR2B, ZMIZ1, ANKRD27, PTP4A1, SPARC, } \\
\text { COL5A1, ZBTB5, CSRNP2, ADAM12, TET3, ITGA6, ENPP2, POU2F2, NAA40, HMGCR, SLC2A14, ZNF507, COL4A1, } \\
\text { RAPGEF2, ENTPD1, FAM71F2, RAP2C, INSIG1, PTEN, ZBTB34, KCTD15, HIST1H2AE, EDC3, REST, DYNLT1, EMP1, } \\
\text { CCSAP, ISG20L2, FBN1, CREB5, BACE1, ZFP91, RPA1, CALM3, FAM84A, REL, CERS2, MAT2A, PDGFC }\end{array}$ \\
\hline $\begin{array}{l}\text { hsa-miR- } \\
451\end{array}$ & 31 & $\begin{array}{l}\text { VEGFA, MAF, POM121C, RAB10, DCBLD2, PRKAR2A, CDKN2D, ANKRD42, VANGL2, FAM83C, OSCAR, SLU7, } \\
\text { ARHGAP18, ADAM22, CBX6, DHCR7, SSBP2, SRP54, RHPN2, GGA3, BZW1, MAP3K1, ZMAT3, MCTS1, OSR1, } \\
\text { ELOVL6, STEAP2, CAB39, PIP4K2C, ZBED2, MIF }\end{array}$ \\
\hline
\end{tabular}

Table 6. Target genes of top 10 downregulated differentially expressed miRNAs

\begin{tabular}{|c|c|c|}
\hline miRNAs & Number & predicted target genes \\
\hline $\begin{array}{l}\text { hsa-miR-1225 } \\
-5 p\end{array}$ & 5 & SEMA4D, DCX, ZNF107, SEMA6D, ASPH \\
\hline hsa-miR-373 & 16 & $\begin{array}{l}\text { HOOK3, EIF4E, TRIM66, PDP2, SUZ12, FGF5, RNPS1, DNAL1, SPIB, SATB2, LIMA1, SGTB, FREM2, RPF2, TTC31, } \\
\text { GTF2IRD2 }\end{array}$ \\
\hline hsa-miR-647 & 8 & NCOA1, ZNF609, NFIX, HNRNPF, SECISBP2L, NIPBL, IL5, MG1 \\
\hline $\begin{array}{l}\text { hsa-miR-483- } \\
3 p\end{array}$ & 4 & IGF1, MAPKAPK2, RAB5B, CNOT6L \\
\hline hsa-miR-485- & 1 & RORA \\
\hline hsa-miR-498 & 69 & $\begin{array}{l}\text { EP300, PPP1R15B, KLHL36, EPHA4, LIN28B, LIMD2, PLEKHM3, GK5, EPB41L3, GALNT10, ELK4, CBX4, ZFP36L1, } \\
\text { UBE2H, C16orf72, MSMO1, PDIA6, ZNF689, IRF2BP2, SFPQ, YOD1, PAG1, MID1, MATN3, ZNF134, KCTD20, } \\
\text { MYO1F, CDV3, DSN1, VTA1, TOB1, ATP2B1, PELI2, IKZF3, PALM2, CRCP, HYPK, SLC7A11, S100A16, SAMD5, } \\
\text { GPR155, FOXJ3, SLC2A14, IL17RD, MEX3A, COL4A1, SERF2, PLEKHG1, LACTB, SCO1, TRDN, DICER1, CAPZA2, } \\
\text { WT1, CDK6, MORF4L2, SYNRG, FRAT2, ZKSCAN4, PDCD10, CHAC1, MCTS1, ASXL3, LOXL2, WIPF2, CYP20A1, } \\
\text { KLHL8, MED28, FAM217B }\end{array}$ \\
\hline
\end{tabular}




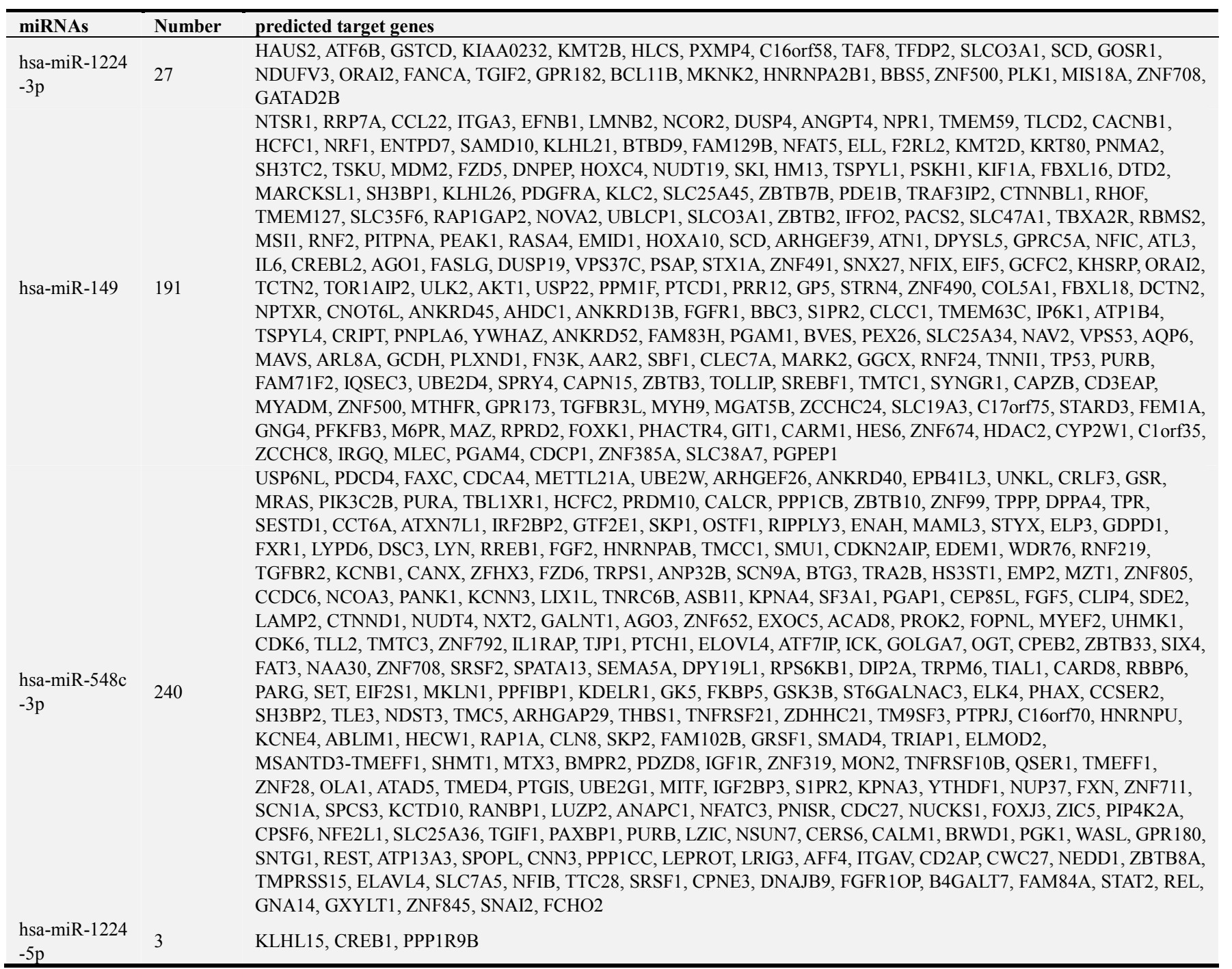

Table 7. Top 20 clusters with their representative enriched terms of upregulated and downregulated DE-genes (one per cluster).

\begin{tabular}{|c|c|c|c|c|c|c|}
\hline GO & Category & Description & Count & $\%$ & $\log 10(P)$ & DE-genes \\
\hline R-HSA-912446 & Reactome Gene Sets & Meiotic recombination & 12 & 4.43 & -9.64 & upregulated \\
\hline GO:0060142 & $\begin{array}{l}\text { GO Biological } \\
\text { Processes }\end{array}$ & $\begin{array}{l}\text { regulation of syncytium formation by plasma membrane } \\
\text { fusion }\end{array}$ & 5 & 1.85 & -4.79 & upregulated \\
\hline R-HSA-5689901 & Reactome Gene Sets & Metalloprotease DUBs & 5 & 1.85 & -4.25 & upregulated \\
\hline hsa04060 & KEGG Pathway & Cytokine-cytokine receptor interaction & 12 & 4.43 & -4.23 & upregulated \\
\hline R-HSA-5688426 & Reactome Gene Sets & Deubiquitination & 12 & 4.43 & -3.84 & upregulated \\
\hline GO:0002476 & $\begin{array}{l}\text { GO Biological } \\
\text { Processes }\end{array}$ & $\begin{array}{l}\text { antigen processing and presentation of endogenous peptide } \\
\text { antigen via MHC class Ib }\end{array}$ & 3 & 1.11 & -3.8 & upregulated \\
\hline R-HSA-202733 & Reactome Gene Sets & Cell surface interactions at the vascular wall & 8 & 2.95 & -3.77 & upregulated \\
\hline GO:0097191 & $\begin{array}{l}\text { GO Biological } \\
\text { Processes }\end{array}$ & extrinsic apoptotic signaling pathway & 10 & 3.69 & -3.63 & upregulated \\
\hline GO:0051549 & $\begin{array}{l}\text { GO Biological } \\
\text { Processes }\end{array}$ & positive regulation of keratinocyte migration & 3 & 1.11 & -3.55 & upregulated \\
\hline GO:0045217 & $\begin{array}{l}\text { GO Biological } \\
\text { Processes }\end{array}$ & cell-cell junction maintenance & 3 & 1.11 & -3.55 & upregulated \\
\hline GO:0030099 & $\begin{array}{l}\text { GO Biological } \\
\text { Processes }\end{array}$ & myeloid cell differentiation & 14 & 5.17 & -3.48 & upregulated \\
\hline GO:0060603 & $\begin{array}{l}\text { GO Biological } \\
\text { Processes }\end{array}$ & mammary gland duct morphogenesis & 4 & 1.48 & -3.36 & upregulated \\
\hline R-HSA-391160 & Reactome Gene Sets & Signal regulatory protein family interactions & 3 & 1.11 & -3.15 & upregulated \\
\hline M5885 & Canonical Pathways & NABA MATRISOME ASSOCIATED & 19 & 7.01 & -3.04 & upregulated \\
\hline GO:0050708 & $\begin{array}{l}\text { GO Biological } \\
\text { Processes }\end{array}$ & regulation of protein secretion & 14 & 5.17 & -2.85 & upregulated \\
\hline R-HSA-69618 & Reactome Gene Sets & Mitotic Spindle Checkpoint & 6 & 2.21 & -2.78 & upregulated \\
\hline
\end{tabular}




\begin{tabular}{|c|c|c|c|c|c|c|}
\hline GO & Category & Description & Count & $\%$ & $\log 10(P)$ & DE-genes \\
\hline GO:0051496 & $\begin{array}{l}\text { GO Biological } \\
\text { Processes }\end{array}$ & positive regulation of stress fiber assembly & 4 & 1.48 & -2.56 & upregulated \\
\hline hsa04144 & KEGG Pathway & Endocytosis & 9 & 3.32 & -2.55 & upregulated \\
\hline GO:0006968 & $\begin{array}{l}\text { GO Biological } \\
\text { Processes }\end{array}$ & cellular defense response & 4 & 1.48 & -2.53 & upregulated \\
\hline GO:0008625 & $\begin{array}{l}\text { GO Biological } \\
\text { Processes }\end{array}$ & $\begin{array}{l}\text { extrinsic apoptotic signaling pathway via death domain } \\
\text { receptors }\end{array}$ & 5 & 1.85 & -2.52 & upregulated \\
\hline GO:0070838 & $\begin{array}{l}\text { GO Biological } \\
\text { Processes }\end{array}$ & divalent metal ion transport & 14 & 6.7 & -3.95 & downregulated \\
\hline GO:0008298 & $\begin{array}{l}\text { GO Biological } \\
\text { Processes }\end{array}$ & intracellular mRNA localization & 3 & 1.44 & -3.88 & downregulated \\
\hline GO:0048820 & $\begin{array}{l}\text { GO Biological } \\
\text { Processes }\end{array}$ & hair follicle maturation & 3 & 1.44 & -3.48 & downregulated \\
\hline GO:0017145 & $\begin{array}{l}\text { GO Biological } \\
\text { Processes }\end{array}$ & stem cell division & 4 & 1.91 & -3.29 & downregulated \\
\hline GO:0070371 & $\begin{array}{l}\text { GO Biological } \\
\text { Processes }\end{array}$ & ERK1 and ERK2 cascade & 10 & 4.78 & -3.22 & downregulated \\
\hline hsa03060 & KEGG Pathway & Protein export & 3 & 1.44 & -3 & downregulated \\
\hline GO:0050727 & $\begin{array}{l}\text { GO Biological } \\
\text { Processes }\end{array}$ & regulation of inflammatory response & 12 & 5.74 & -2.78 & downregulated \\
\hline R-HSA-5218920 & Reactome Gene Sets & VEGFR2 mediated vascular permeability & 3 & 1.44 & -2.7 & downregulated \\
\hline GO:1901661 & $\begin{array}{l}\text { GO Biological } \\
\text { Processes }\end{array}$ & quinone metabolic process & 3 & 1.44 & -2.54 & downregulated \\
\hline R-HSA-418594 & Reactome Gene Sets & $\mathrm{G}$ alpha (i) signalling events & 10 & 4.78 & -2.52 & downregulated \\
\hline GO:0019228 & $\begin{array}{l}\text { GO Biological } \\
\text { Processes }\end{array}$ & neuronal action potential & 3 & 1.44 & -2.5 & downregulated \\
\hline GO:0060047 & $\begin{array}{l}\text { GO Biological } \\
\text { Processes }\end{array}$ & heart contraction & 8 & 3.83 & -2.47 & downregulated \\
\hline GO:0001525 & $\begin{array}{l}\text { GO Biological } \\
\text { Processes }\end{array}$ & angiogenesis & 12 & 5.74 & -2.24 & downregulated \\
\hline GO:0001667 & $\begin{array}{l}\text { GO Biological } \\
\text { Processes }\end{array}$ & ameboidal-type cell migration & 10 & 4.78 & -2.08 & downregulated \\
\hline GO:0030029 & $\begin{array}{l}\text { GO Biological } \\
\text { Processes }\end{array}$ & actin filament-based process & 14 & 6.7 & -2.06 & downregulated \\
\hline GO:0034620 & $\begin{array}{l}\text { GO Biological } \\
\text { Processes }\end{array}$ & cellular response to unfolded protein & 5 & 2.39 & -2.06 & downregulated \\
\hline GO:0060711 & $\begin{array}{l}\text { GO Biological } \\
\text { Processes }\end{array}$ & labyrinthine layer development & 3 & 1.44 & -2.06 & downregulated \\
\hline
\end{tabular}

"Count" is the number of genes in the user-provided lists with membership in the given ontology term. "\%" is the percentage of all of the user-provided genes that are found in the given ontology term (only input genes with at least one ontology term annotation were included in the calculation). "Log10 (P)" is the p-value in $\log$ base $10 . " \log 10(\mathrm{q}) "$ is the multi-test adjusted p-value in $\log$ base $10 . \mathrm{DE}=$ differentially expressed.

Table 8. Top 20 clusters with their representative enriched terms of downregulated and upregulated DE-miRNAs (one per cluster).

\begin{tabular}{|c|c|c|c|c|c|c|}
\hline GO & Category & Description & Count & $\%$ & $\log 10(P)$ & DE-miRNAs \\
\hline O:0016569 & GO Biological Processes & covalent chromatin modification & 33 & 6.04 & -7.79 & downregulated \\
\hline hsa05200 & KEGG Pathway & Pathways in cancer & 28 & 5.13 & -6.93 & downregulated \\
\hline R-HSA-9006925 & Reactome Gene Sets & Intracellular signaling by second messengers & 24 & 4.4 & -6.83 & downregulated \\
\hline GO:0034248 & GO Biological Processes & regulation of cellular amide metabolic process & 32 & 5.86 & -6.7 & downregulated \\
\hline GO:0006476 & GO Biological Processes & protein deacetylation & 13 & 2.38 & -6.6 & downregulated \\
\hline GO:0006403 & GO Biological Processes & RNA localization & 20 & 3.66 & -6.32 & downregulated \\
\hline R-HSA-9022692 & Reactome Gene Sets & Regulation of MECP2 expression and activity & 8 & 1.47 & -6.26 & downregulated \\
\hline GO:2000147 & GO Biological Processes & positive regulation of cell motility & 34 & 6.23 & -6.24 & downregulated \\
\hline GO:0044380 & GO Biological Processes & protein localization to cytoskeleton & 10 & 1.83 & -6.2 & downregulated \\
\hline hsa04110 & KEGG Pathway & Cell cycle & 14 & 2.56 & -6.07 & downregulated \\
\hline GO:0010498 & GO Biological Processes & proteasomal protein catabolic process & 29 & 5.31 & -5.73 & downregulated \\
\hline R-HSA-1368082 & Reactome Gene Sets & RORA activates gene expression & 6 & 1.1 & -5.72 & downregulated \\
\hline GO:0045600 & GO Biological Processes & positive regulation of fat cell differentiation & 10 & 1.83 & -5.48 & downregulated \\
\hline GO:0031647 & GO Biological Processes & regulation of protein stability & 21 & 3.85 & -5.47 & downregulated \\
\hline hsa04520 & KEGG Pathway & Adherens junction & 10 & 1.83 & -5.31 & downregulated \\
\hline GO:0010256 & GO Biological Processes & endomembrane system organization & 27 & 4.95 & -5.28 & downregulated \\
\hline GO:0061061 & GO Biological Processes & muscle structure development & 35 & 6.41 & -5.26 & downregulated \\
\hline R-HSA-170834 & Reactome Gene Sets & Signaling by TGF-beta Receptor Complex & 10 & 1.83 & -5.26 & downregulated \\
\hline GO:1903827 & GO Biological Processes & regulation of cellular protein localization & 30 & 5.49 & -5.24 & downregulated \\
\hline GO:0048598 & GO Biological Processes & embryonic morphogenesis & 32 & 5.86 & -5.12 & downregulated \\
\hline GO:0001568 & GO Biological Processes & blood vessel development & 91 & 9.01 & -18.31 & upregulated \\
\hline GO:0007265 & GO Biological Processes & Ras protein signal transduction & 53 & 5.25 & -12.86 & upregulated \\
\hline
\end{tabular}




\begin{tabular}{|c|c|c|c|c|c|c|}
\hline GO & Category & Description & Count & $\%$ & $\log 10(P)$ & DE-miRNAs \\
\hline GO:0007507 & GO Biological Processes & heart development & 78 & 7.72 & -18.84 & upregulated \\
\hline GO:0008285 & GO Biological Processes & negative regulation of cell proliferation & 83 & 8.22 & -14.5 & upregulated \\
\hline GO:0009896 & GO Biological Processes & positive regulation of catabolic process & 55 & 5.45 & -12.53 & upregulated \\
\hline GO:0010638 & GO Biological Processes & positive regulation of organelle organization & 72 & 7.13 & -13.61 & upregulated \\
\hline GO:0030099 & GO Biological Processes & myeloid cell differentiation & 54 & 5.35 & -12.52 & upregulated \\
\hline GO:0030335 & GO Biological Processes & positive regulation of cell migration & 70 & 6.93 & -15.6 & upregulated \\
\hline GO:0045596 & GO Biological Processes & negative regulation of cell differentiation & 79 & 7.82 & -13.09 & upregulated \\
\hline GO:0045786 & GO Biological Processes & negative regulation of cell cycle & 70 & 6.93 & -12.78 & upregulated \\
\hline GO:0048589 & GO Biological Processes & developmental growth & 73 & 7.23 & -12.79 & upregulated \\
\hline GO:0061061 & GO Biological Processes & muscle structure development & 82 & 8.12 & -17.43 & upregulated \\
\hline GO:0070848 & GO Biological Processes & response to growth factor & 84 & 8.32 & -16.23 & upregulated \\
\hline GO:0071407 & GO Biological Processes & cellular response to organic cyclic compound & 64 & 6.34 & -13.07 & upregulated \\
\hline GO:0071900 & GO Biological Processes & regulation of protein serine/threonine kinase activity & 66 & 6.53 & -14.74 & upregulated \\
\hline GO:0080135 & GO Biological Processes & regulation of cellular response to stress & 82 & 8.12 & -14.29 & upregulated \\
\hline R-HSA-5663202 & Reactome Gene Sets & $\begin{array}{l}\text { Diseases of signal transduction by growth factor } \\
\text { receptors and second messengers }\end{array}$ & 52 & 5.15 & -13.52 & upregulated \\
\hline R-HSA-9006925 & Reactome Gene Sets & Intracellular signaling by second messengers & 44 & 4.36 & -12.08 & upregulated \\
\hline hsa05200 & KEGG Pathway & Pathways in cancer & 59 & 5.84 & -16.79 & upregulated \\
\hline hsa05206 & KEGG Pathway & MicroRNAs in cancer & 43 & 4.26 & -11.82 & upregulated \\
\hline
\end{tabular}

"Count" is the number of genes in the user-provided lists with membership in the given ontology term. "\%" is the percentage of all of the user-provided genes that are found in the given ontology term (only input genes with at least one ontology term annotation are included in the calculation). "Log10 (P)" is the p-value in $\log$ base 10. "Log10 (q)" is the multi-test adjusted p-value in log base 10

$\mathrm{DE}=$ differentially expressed.

\section{References}

[1] P. B. Allen, Mary Jo Lechowicz, Management of NK/T-Cell Lymphoma, Nasal Type, Journal of Oncology Practice 15 (10) (2019) 513-520.

[2] S. V. Adams, P. A. Newcomb, A. R. Shustov, Racial patterns of peripheral T-cell lymphoma incidence and survival in the united states, Journal of clinical oncology 34 (9) (2016) 963-971.

[3] M. Yamaguchi, R. Suzuki, M. Oguchi, Advances in the treatment of extranodal NK/T-cell lymphoma, nasal type, Blood 131 (23) (2018) 2528-2540.

[4] N. Somasundaram, J. Q. Lim, C. K. Ong, S. T. Lim, Pathogenesis and biomarkers of natural killer T cell lymphoma (NKTL), J Hematol Oncol 12 (1) (2019) 28.

[5] A. C. Mallory, H. Vaucheret, MicroRNAs: something important between the genes, Current Opinion in Plant Biology 7 (2) (2004) 120-125.

[6] S. P. Kabekkodu, V. Shukla, V. K. Varghese, J. D. Souza, S. Chakrabarty, K. Satyamoorthy, Clustered miRNAs and their role in biological functions and diseases, Biological Reviews Cambridge Philosophical Society 93 (4) (2018) 1955-1986.

[7] J. Yan, B. Li, B. Lin, P. T. Lee, T.-H. Chung, J. Tan, C. Bi, X. T. Lee, V. Selvarajan, S.-B. Ng, H. Yang, Q. Yu, W.-J. Chng, EZH2 phosphorylation by JAK3 mediates a switch to noncanonical function in natural killer/T-cell lymphoma, Blood 128 (7) (2016) 948-958.

[8] S.-B. Ng, J. Yan, G. Huang, V. Selvarajan, J. L.-S. Tay, B. Lin, C. Bi, J. Tan, Y.-L. Kwong, N. Shimizu, K. Aozasa, W.-J. Chng, Dysregulated microRNAs affect pathways and targets of biologic relevance in nasal-type natural killer/T-cell lymphoma, Blood 118 (18) (2011) 4919-4929.

[9] V. Agarwal, G. W. Bell, J.-W. Nam, D. P. Bartel, Predicting effective microRNA target sites in mammalian mRNAs, Elife 4
(2015) e05005.

[10] S.-D. Hsu, F.-M. Lin, W.-Y. Wu, C. Liang, W.-C. Huang, W.-L. Chan, W.-T. Tsai, G.-Z. Chen, C.-J. Lee, C.-M. Chiu, C.-H. Chien, M.-C. Wu, C.-Y. Huang, A.-P. Tsou, H.-D. Huang, miRTarBase: a database curates experimentally validated microRNA-target interactions, Nucleic Acids Research 39 (Database issue) (2011) D163-D169.

[11] Y. Chen, X. Wang, miRDB: an online database for prediction of functional microRNA targets, Nucleic Acids Research 48 (D1) (2020) D127-D131.

[12] Y. Zhou, B. Zhou, L. Pache, M. Chang, A. H. Khodabakhshi, O. Tanaseichuk, C. Benner, S. K. Chanda, Metascape provides a biologist-oriented resource for the analysis of systems-level datasets, Nature Communications 10 (1) (2019) 1523.

[13] J. Zhang, M. Gurusaran, Y. Fujiwara, K. Zhang, M. Echbarthi, E. Vorontsov, R. Guo, D. F. Pendlebury, I. Alam, G. Livera, M. Emmanuelle, P. J. Wang, J. Nandakumar, O. R. Davies, H. Shibuya, The BRCA2-MEILB2-BRME1 complex governs meiotic recombination and impairs the mitotic BRCA2-RAD51 function in cancer cells, Nature Communications 11 (1) (2020) 2055.

[14] S. de Mel, S. S. Hue, A. D. Jeyasekharan, W. J. Chng, S. B. Ng, Molecular pathogenic pathways in extranodal NK/T cell lymphoma, J Hematol Oncol 12 (1) (2019) 33.

[15] S. Zhang, J. Yuan, R. Zheng, Suppression of ubiquitin-specific peptidase 17 (USP17) inhibits tumorigenesis and invasion in non-small cell lung cancer cells, Oncology Research 24 (4) (2016) 263-269.

[16] Y. Song, W. Song, Zhaoming, W. Song, Y. Wen, J. Li, Q. Xia, M. Zhang, CDC27 promotes tumor progression and affects PD-L1 expression in T-Cell lymphoblastic lymphoma, Frontiers in Oncology 10 (2020) 488.

[17] X. Zhao, Y. Lei, G. Li, Y. Cheng, H. Yang, L. Xie, H. Long, R. Jiang, Integrative analysis of cancer driver genes in prostate adenocarcinoma, Molecular Medicine Reports 19 (4) (2019) 2707-2715. 
[18] M. Vidak, I. Jovcevska, N. Samec, A. Zottel, M. Liovic, D. Rozman, S. Dzeroski, P. Juvan, R. Komel, Meta-analysis and experimental validation identified FREM2 and SPRY1 as new glioblastoma marker candidates, International Journal of Molecular Sciences 19 (5) (2018) 1369.

[19] T. Tanaka, M. Arai, S. Wu, T. Kanda, H. Miyauchi, F. Imazeki, H. Matsubara, O. Yokosuka, Epigenetic silencing of microRNA-373 plays an important role in regulating cell proliferation in colon cancer, Oncology Reports 26 (5) (2011) 1329-1335.

[20] X. Ju, D. Li, Q. Shi, H. Hou, N. Sun, B. Shen, Differential microRNA expression in childhood B-cell precursor acute lymphoblastic leukemia, Pediatric Hematology and Oncology 26 (1) (2009) 1-10.

[21] M. Schraders, P. Jares, S. Bea, E. F. P. M. Schoenmakers, J. H. J. M. v. Krieken, E. Campo, P. J. T. A. Groenen, Integrated genomic and expression profiling in mantle cell lymphoma: identification of gene-dosage regulated candidate genes, British Journal Haematology 143 (2) (2008) 210-221.

[22] Y. Wang, J. Huang, Y. Ma, G. Tang, Y. Liu, X. Chen, Z. Zhang, L. Zeng, Y. Wang, Y. B. Ouyang, G. Y. Yang, MicroRNA-29b is a therapeutic target in cerebral ischemia associated with aquaporin 4, J Cereb Blood Flow Metab 35 (12) (2015) 1977-84.

[23] L. Diamanti, D. Franciotta, G. Berzero, P. Bini, L. Farina, A. Colombo, M. Ceroni, E. Marchioni, Late post-transplant anti-aquaporin-4 Ab-positive optic neuritis in a patient with AML, Bone Marrow Transplantation 50 (8) (2015) 1125-1126.

[24] L. Simone, F. Pisani, M. G. Mola, M. D. Bellis, G. Merla, L. Micale, A. Frigeri, A. L. Vescovi, M. Svelto, G. P. Nicchia, AQP4 aggregation state is a determinant for glioma cell fate, Cancer Research 79 (9) (2019) 2182-2194.

[25] R. Garzon, S. Liu, M. Fabbri, Z. Liu, C. E. A. Heaphy, E.
Callegari, S. Schwind, J. Pang, J. Yu, N. Muthusamy, V. Havelange, S. Volinia, W. Blum, L. J. Rush, D. Perrotti, M. Andreeff, C. D. Bloomfield, J. C. Byrd, K. Chan, L.-C. Wu, C. M. Croce, G. Marcucci1, MicroRNA-29b induces global DNA hypomethylation and tumor suppressor gene reexpression in acute myeloid leukemia by targeting directly DNMT3A and 3B and indirectly DNMT1, Blood 113 (25) (2009) 6411-6418.

[26] R. Kohnken, J. Wen, B. Mundy-Bosse, K. McConnell, A. Keiter, L. Grinshpun, A. Hartlage, M. Yano, B. McNeil, N. Chakravarti, B. William, J. E. Bradner, M. A. Caligiuri, P. Porcu, A. Mishra, Diminished microRNA-29b level is associated with BRD4-mediated activation of oncogenes in cutaneous T-cell lymphoma, Blood 131 (7) (2018) 771-781.

[27] L. Mazzoccoli, M. C. Robaina, A. G. Apa, M. Bonamino, L. W. Pinto, E. Queiroga, C. E. Bacchi, C. E. Klumb, MiR-29 silencing modulates the expression of target genes related to proliferation, apoptosis and methylation in Burkitt lymphoma cells, Journal of Cancer Research and Clinical Oncology 144 (3) (2018) 483-497.

[28] M. Vidak, I. Jovcevska, N. Samec, A. Zottel, M. Liovic, D. Rozman, S. Dzeroski, P. Juvan, R. Komel, miR-29b suppresses tumor growth and metastasis in colorectal cancer via downregulating Tiam1 expression and inhibiting epithelial-mesenchymal transition, Cell Death and Disease 5 (2014) e1335.

[29] E. Iwanaga, T. Nanri, H. Mitsuya, N. Asou, Mutation in the RNA binding protein TIS11D/ZFP36L2 is associated with the pathogenesis of acute leukemia, International journal of oncology 38 (1) (2011) 25-31.

[30] D. J. Hodson, M. L. Janas, A. Galloway, S. E. Bell, S. Andrews, C. M. Li1, R. Pannell, C. W. Siebel, H. R. MacDonald, K. D. Keersmaecker, A. A. Ferrando, G. Grutz, M. Turner, Deletion of the RNA-binding proteins ZFP36L1 and ZFP36L2 leads to perturbed thymic development and T lymphoblastic leukemia, Nature immunology 11 (8) (2010) 717-724. 